\title{
A hypoplastic model for gas hydrate-bearing sandy sediments
}

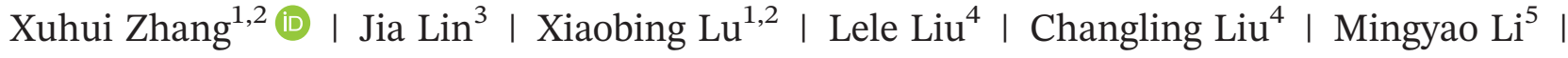 \\ Yewang $\mathrm{Su}^{5,2,6}$
}

${ }^{1}$ Key Laboratory for Mechanics in Fluid Solid Coupling Systems, Institute of Mechanics, Chinese Academy of Sciences, Beijing 100190, China

${ }^{2}$ School of Engineering Science, University of Chinese Academy of Sciences, Beijing 100049, China

${ }^{3}$ University of Natural Resources and Life Sciences, Vienna, Austria

${ }^{4}$ Qingdao Institute of Marine Geology, Qingdao, China

${ }^{5}$ State Key Laboratory of Nonlinear Mechanics, Institute of Mechanics, Chinese Academy of Sciences, Beijing 100190, China

${ }^{6}$ State Key laboratory of Hydroscience and Engineering, Tsinghua University, Beijing 100084, China

\section{Correspondence}

X. H. Zhang, Key Laboratory for Mechanics in Fluid Solid Coupling Systems, Institute of Mechanics, Chinese Academy of Sciences, Beijing 100190, China.

Email: zhangxuhui@imech.ac.cn

\section{Funding information}

the National Natural Science Foundation in China, Grant/Award Numbers: 41376078 and 51639008, 51639008 and 41376078; Youth Innovation Promotion Association CAS; State Key Laboratory of Hydroscience and Engineering, Tsinghua University, Grant/Award Number: sklhse2017-D-02; China Geological Survey, Grant/Award Number: DD20160216

\begin{abstract}
Summary
Gas hydrate-bearing sediments (GHBSs) have been considered as a potential energy resource. In this paper, the mechanical properties of GHBS are firstly investigated by the integrated test apparatus for synthesis of GHBS using silty sand as skeleton. Triaxial tests indicate an obvious transition of stress-strain relationship from strain hardening under low hydrate saturation and strain softening under high hydrate saturation. The hypoplastic models coupled with Drucker-Prager criterion and the Mohr-Coulomb criterion are proposed to analyze the stress-strain relationship of GHBS with considering the effective porosity because of the hydrate filling in the pores of GHBS. The strain hardening and softening behaviors are well predicted with less material parameters compared with the classical models. Compared with the test results, the proposed hypoplastic models are verified to be capable of capturing the salient features of the mechanical behaviors of GHBS under the conditions of little temperature change and no hydrate dissociation.
\end{abstract}

\section{KEYWORDS}

effective porosity, gas hydrate-bearing sediment, hypo-plastic model, strain hardening, strain softening

\section{1 | INTRODUCTION}

As the most common naturally occurring gas hydrate, gas hydrate-bearing sediments (GHBSs) have been considered as a potential energy resource, and the related exploration and exploitation technologies have been extensively investigated in many countries. Gas hydrate-bearing sediment, a crystalline solid composed of natural gas and hydrogen-bonded water 
molecules, is typically formed under the relatively low temperature and high pressure conditions and naturally exists in the permafrost regions and continental offshore worldwide. ${ }^{1}$ However, the stability and behaviors of GHBS are strongly dependent on thermohydromechanical conditions or human activities during the extraction, and the dissociation of hydrates may cause massive seafloor instabilities and engineering geological problems, such as submarine landslide, borehole instability, and foundation failures. The proper understanding of the behavior of GHBS related to hydrate formation and dissociation is significant to the drilling and production applications of the large amount of the potential energy resource. ${ }^{2-4}$

The laboratory studies have been comprehensively conducted using reconstituted samples and focus on triaxial compression tests to investigate the mechanical properties of GHBS. The behavior of GHBS is complex in natural and the effects of hydrate saturation, temperature, and effective confining pressure on the modulus and the shear strength have been studied mainly by using reconstituted hydrate-bearing sands. ${ }^{1,5-7}$ The experimental data show that with the increase of hydrate saturation and effective confining pressure, the elastic modulus and shear strength are generally increased. Strain softening occurs at high hydrate saturation (typically about 25\% to 45\%) while plastic and strain hardening is observed at low hydrate saturation. ${ }^{1,8}$ The temperature below freezing point leads to the formation of frozen GHBS (composed of ice, hydrate, and silt sand), and the mechanical behavior strengthens with the increase of the total saturation of hydrate and ice. ${ }^{9}$ The tetrahydrofuran hydrate-bearing silt clay shows typical ductile behavior, and the shear strength increases linearly with the increase of hydrate saturation and confining pressure. ${ }^{10}$

Several models have been proposed to formulate the stress-strain relationship of GHBS. ${ }^{11,12}$ The elastic modulus of gas hydrate-bearing coarse sand is formulated by series and parallel model, ${ }^{13}$ and the shear strength of tetrahydrofuran hydrate-bearing clay is analyzed on the basis of the Mohr-Coulomb and Drucker-Prager criterion. ${ }^{10}$ The Duncan-Chang model is applied to simulate the nonlinear elastic behavior of GHBS. ${ }^{14-16}$ The distinct element model is adopted to describe the cohesion and friction between grains and hydrate in GHBS. ${ }^{17}$ The modified Cam-Clay model is used to take into account the shearing and volumetric dilation of hydrate-bearing sands. The temperature and hydrate saturation can be introduced into these models; however, the number of the material parameters increase, and the strain softening and dilatant behavior of GHBS are still difficult to take into account at the same time.

In most of the abovementioned constitutive models, it is assumed that the deformation of GHBS is infinitesimal, and the strain can be decomposed into elastic and plastic parts and modeled according to the theory of elastoplasticity. For the purpose of simple and practical in the engineering applications, a hypoplastic model is proposed to describe the 3dimensional nonlinear stress-strain behaviors of GHBS. Taking advantage of the hypoplastic model, it is not necessary to consider the yield surface, plastic potential, flow and hardening rules, and decomposition of deformation. The hypoplastic model is based on the nonlinear tensor functions and is capable of capturing the salient features of granular materials, in particular for the problems involving extremely low stress or extremely high stress levels, ${ }^{18-21}$ while the hypoplastic model does not take into account the coupling between mechanics, fluid, and heat.

Because the mechanical behavior of unsaturated GHBS is investigated under the conditions of little temperature change and no hydrate dissociation, the coupling between mechanics, fluid, and heat is not considered. The objective of this paper is to present a hypoplastic model of GHBS, understand the mechanism of strain softening at high hydrate saturation, and validate the model by experiments capturing the main composition and geotechnical condition of natural GHBS. Firstly, hypoplastic models are recapitulated within the theoretical framework of hypoplasticity and proposed to formulate the mechanical behaviors of GHBS on the basis of experimental investigation. The shear strength is modeled on the basis of the modified Drucker-Prager criterion and Mohr-Coulomb criterion, respectively. The effects of critical void ratio and effective porosity under different hydrate saturations are taking into account in the proposed hypoplastic model to describe the strain softening behavior. Secondly, the experimental apparatus and procedures are fully presented, and a set of triaxial compression tests are conducted on reconstituted GHBS by using silt sand as skeleton under various hydrate saturations and effective confining pressures. Finally, the proposed model is applied to describe the strain hardening and softening behaviors and compared with the experimental results under various hydrate saturations and confining pressures.

\section{2 | A HYPOPLASTIC MODEL FOR GHBS}

As an alternative approach to the general elastoplastic models, a simple hypoplastic constitutive model is proposed to describe the mechanical behavior of GHBS as follows ${ }^{20}$ :

$$
\dot{\sigma}=c_{1}(\operatorname{tr} \sigma) \dot{\varepsilon}+c_{2} \frac{\operatorname{tr}(\sigma \dot{\varepsilon}) \sigma}{\operatorname{tr} \sigma}+\left(c_{3} \frac{\sigma^{2}}{\operatorname{tr} \sigma}+c_{4} \frac{\left(\sigma^{*}\right)^{2}}{\operatorname{tr} \sigma}\right)\|\dot{\varepsilon}\|,
$$


where $\dot{\sigma}$ and $\dot{\varepsilon}$ are the rates of stress and strain tensors; $\|\dot{\varepsilon}\|=\sqrt{\operatorname{tr} \dot{\varepsilon}^{2}}$ denotes the Euclidean norm; and $c_{1}, c_{2}, c_{3}, c_{4}$ are dimensionless material parameters, which are independent of the invariants and joint invariants of $\sigma$ and $\dot{\varepsilon}$. The deviatoric stress $\sigma^{*}$ is defined as

$$
\sigma^{*}=\sigma-\frac{1}{3}(\operatorname{tr} \sigma) \boldsymbol{I}
$$

where $\sigma$ is the stress tensor and $\boldsymbol{I}$ is the unit tensor.

To obtain a feasible constitutive equation, the parameters are required to be calibrated according to largely various laboratory tests. To this end, we chose the most widely used triaxial tests, and the matrices of $\sigma, \dot{\varepsilon}$, and $\dot{\sigma}$ can be expressed as follows:

$$
\boldsymbol{\sigma}=\left[\begin{array}{ccc}
\sigma_{1} & 0 & 0 \\
0 & \sigma_{2} & 0 \\
0 & 0 & \sigma_{3}
\end{array}\right], \dot{\varepsilon}=\left[\begin{array}{ccc}
\dot{\varepsilon}_{1} & 0 & 0 \\
0 & \dot{\varepsilon}_{2} & 0 \\
0 & 0 & \dot{\varepsilon}_{3}
\end{array}\right], \dot{\sigma}=\left[\begin{array}{ccc}
\dot{\sigma}_{1} & 0 & 0 \\
0 & \dot{\sigma}_{2} & 0 \\
0 & 0 & \dot{\sigma}_{3}
\end{array}\right]
$$

The 4 coefficients of constitutive equation proposed in Equation 1 can then be determined by substituting the corresponding matrices according to the triaxial compression tests. The differential equations are obtained as follows:

$$
\begin{aligned}
& \dot{\sigma}_{1}=c_{1}\left(\sigma_{1}+2 \sigma_{3}\right) \dot{\varepsilon}_{1}+c_{2} \frac{\sigma_{1} \dot{\varepsilon}_{1}+2 \sigma_{3} \dot{\varepsilon}_{3}}{\sigma_{1}+2 \sigma_{3}} \sigma_{1}+\left[c_{3} \sigma_{1}^{2}+\frac{4}{9} c_{4}\left(\sigma_{1}-\sigma_{3}\right)^{2}\right] \frac{\sqrt{\dot{\varepsilon}_{1}^{2}+2 \dot{\varepsilon}_{3}^{2}}}{\sigma_{1}+2 \sigma_{3}} \\
& \dot{\sigma}_{3}=c_{1}\left(\sigma_{1}+2 \sigma_{3}\right) \dot{\varepsilon}_{3}+c_{2} \frac{\sigma_{1} \dot{\varepsilon}_{1}+2 \sigma_{3} \dot{\varepsilon}_{3}}{\sigma_{1}+2 \sigma_{3}} \sigma_{3}+\left[c_{3} \sigma_{3}^{2}+\frac{4}{9} c_{4}\left(\sigma_{1}-\sigma_{3}\right)^{2}\right] \frac{\sqrt{\dot{\varepsilon}_{1}^{2}+2 \dot{\varepsilon}_{3}^{2}}}{\sigma_{1}+2 \sigma_{3}}
\end{aligned}
$$

The above 2 equations are 2 linear ones with 4 unknown material parameters $\left(c_{1}, c_{2}, c_{3}, c_{4}\right)$, which can be obtained by solving the equation system. To this end, the 4 parameters can be related to some well-established parameters in soil mechanics by considering some specific stress states, for example, the hydrostatic and the stress state at failure. The detail information is referred to the work of Wu and Bauer, ${ }^{20}$ and the explicit expressions of $c_{1}, c_{2}, c_{3}, c_{4}$ are given as

$$
\begin{aligned}
& c_{1}=\frac{E_{i}}{3 \sigma_{3}\left(1+v_{i}\right)}, \\
& c_{2}=\frac{9 d_{f} v_{i}\left(R_{f}^{2}-4\right)+d_{i}\left(2+R_{f}\right)^{2}\left(1+4 v_{f}\right)}{d_{f}\left(1-2 v_{i}\right)\left(R_{f}^{2}-4\right)+d_{i}\left(2 v_{f}-R_{f}\right)\left(R_{f}-4\right)} c_{1}, \\
& c_{3}=\frac{9 v_{i}\left(2 v_{f}-R_{f}\right)\left(R_{f}-4\right)-\left(1-2 v_{i}\right)\left(2+R_{f}\right)^{2}\left(1+4 v_{f}\right)}{d_{f}\left(1-2 v_{i}\right)\left(R_{f}^{2}-4\right)+d_{i}\left(2 v_{f}-R_{f}\right)\left(R_{f}-4\right)} c_{1}, \\
& c_{4}=\frac{9\left[\left(R_{f}^{2} v_{f}+1\right)\left(2+R_{f}\right)^{2} c_{1}+\left(R_{f}-1\right) R_{f}\left(2 v_{f}-R_{f}\right) c_{2}\right]}{d_{f}\left(R_{f}-1\right)^{2}\left(R_{f}^{2}-4\right)},
\end{aligned}
$$

where the initial tangent modulus $E_{i}=\left[\left(\dot{\sigma}_{1}-\dot{\sigma}_{3}\right) / \dot{\varepsilon}_{1}\right]_{R=1}$, the initial Poisson ratio $v_{i}=\left[\dot{\varepsilon}_{3} / \dot{\varepsilon}_{1}\right]_{R=1}$, the failure stress ratio $R_{f}=\left[\sigma_{1} / \sigma_{3}\right]_{\max }$, and the failure ratio $v_{f}=\left(\dot{\varepsilon}_{3} / \dot{\varepsilon}_{1}\right)_{R=R f}$, in which $R=\sigma_{1} / \sigma_{3}$ being the stress ratio with $d_{i}=\sqrt{1+2 v_{i}^{2}}$ and $d_{f}=\sqrt{1+2 v_{f}^{2}}$.

For the purpose of simplicity in engineering practice, the former expressions can be further simplified in view of the triaxial compression tests. Extensive experiments on various granular materials have verified that the radial strain at the initiation of triaxial compression tests can be regarded as zero, ie, $v_{i}=0 .{ }^{20}$ Hence, the expression in Equation 6 can be simplified as 


$$
\begin{aligned}
& c_{1}=\frac{E_{i}}{3 \sigma_{3}}, \\
& c_{2}=c_{3}=\frac{\left(R_{f}+2\right)^{2}\left(1+4 v_{f}\right)}{d_{f}\left(R_{f}^{2}-4\right)+\left(2 v_{f}-R_{f}\right)\left(R_{f}-4\right)} c_{1}, \\
& c_{4}=-\frac{9\left(2+R_{f}\right)^{2}\left[R_{f}^{2} d_{f} v_{f}+R_{f} v_{f}\left(2 v_{f}-R_{f}\right)+d_{f}+\left(2 v_{f}-R_{f}\right)\right]}{d_{f}\left(R_{f}-1\right)^{2}\left[d_{f}\left(R_{f}^{2}-4\right)+\left(2 v_{f}-R_{f}\right)\left(R_{f}-4\right)\right]} c_{1} .
\end{aligned}
$$

As long as $c_{1}$ is determined by fitting the initial tangent modulus of a triaxial compression test, other parameters can be then identified by the failure stress ratio $R_{f}$ and the failure Poisson ratio $v_{f}$ accordingly, such as the Drucker-Prager criterion and Mohr-Coulomb criterion. It is noted that the 4 material coefficients can also be calibrated with other sort of tests and more refined identification can be obtained.

To this end, the constitutive model can be expressed explicitly for a specific kind of materials with the former procedure. Now, the stress can be then updated for a time step $\Delta t$ by integrating the calibrated equations 4 and 5 to get the initial stress for the next time step as follows:

$$
\sigma(t+\Delta t)=\sigma(t)+\int_{t}^{t+\Delta t} \dot{\sigma}[\sigma(\eta), \dot{\varepsilon}(\eta)] d \eta .
$$

To solve the above integral equation, numerical methods are required instead of the analytical solution and a simple 1-step, Euler forward scheme is applied in this paper.

At this point, the entire hypoplastic constitutive model with only 4 material parameters is well achieved, and it is worth noting that many conventional elastoplastic concepts in soil mechanics can be related to this constitutive model. As stated above, the 4 material parameters can be identified by fitting of largely experimental data coupling with some failure criterion, for instance, the Drucker-Prager criterion and Mohr-Coulomb criterion. As is known that the shear strength of the DHBS is strongly affected by degrees of hydrate saturation, the modified Drucker-Prager criterion and the Mohr-Coulomb criterion are adopted by considering the hydrate saturation. The modified Drucker-Prager criterion and the Mohr-Coulomb criterion can introduce the hydrate saturation into the material parameters and describe the shear strength well in the first-order approximation. It is noted that the failure criterion of the Drucker-Prager and the Mohr-Coulomb is equivalent if the yield line of the Drucker-Prager criterion is internally tangent to that of the Mohr-Coulomb criterion in the deviatoric plane under the plain strain condition. ${ }^{10}$

\section{1 | The shear strength modeled by the Drucker-Prager criterion (Model I)}

Taking into account the effects of hydrate saturation, the Drucker-Prager criterion takes the modified form as

$$
\sqrt{J_{2}}=K_{f}\left(S_{h}\right)+\beta\left(S_{h}\right) \cdot I_{1}
$$

where $J_{2}=\left[\left(\sigma_{1}-\sigma_{2}\right)^{2}+\left(\sigma_{2}-\sigma_{3}\right)^{2}+\left(\sigma_{3}-\sigma_{1}\right)^{2}\right] / 6, I_{1}=\sigma_{1}+\sigma_{2}+\sigma_{3}$, the material parameters $K_{f}\left(S_{h}\right)$ and $\beta\left(S_{h}\right)$ are the functions of hydrate saturation $S_{h}$. For the case of triaxial compression tests, the second principle stress equals to the third principle stress, ie, $\sigma_{2}=\sigma_{3}$, and the modified Drucker-Prager criterion can be simplified as

$$
\sigma_{1}-\sigma_{3}=\sqrt{3} K_{f}\left(S_{h}\right)+\sqrt{3} \beta\left(S_{h}\right) \cdot\left(\sigma_{1}+2 \sigma_{3}\right) .
$$

The form can be further expressed as

$$
\sigma_{1}-\sigma_{3}=A\left(S_{h}\right)+B\left(S_{h}\right) \cdot \sigma_{3},
$$

where $A\left(S_{h}\right)=\frac{\sqrt{3} K_{f}\left(S_{h}\right)}{1-\sqrt{3} \beta\left(S_{h}\right)}, B\left(S_{h}\right)=\frac{3 \sqrt{3} \beta\left(S_{h}\right)}{1-\sqrt{3} \beta\left(S_{h}\right)}$.

As taking the first-order approximation, Equation 11 can be simplified as

$$
\sigma_{1}-\sigma_{3}=\left(A^{\prime}(0) \cdot S_{h}+A(0)\right)+\left(B^{\prime}(0) \cdot S_{h}+B(0)\right) \cdot \sigma_{3},
$$

where $A^{\prime}(0)=\partial A\left(S_{h}=0\right) / \partial S_{h}, B^{\prime}(0)=\partial B\left(S_{h}=0\right) / \partial S_{h} \cdot$ 
Following the work of Rowe $\mathrm{e}^{22}$ for the relationship between stress and dilatancy of granular materials under undrained and static loading condition, the ratio of the lateral strain to axial strain, ie, $\dot{\varepsilon}_{3} / \dot{\varepsilon}_{1}$, can be derived from the ratio of axial stress to lateral stress, ie, $\sigma_{1} / \sigma_{3}$, and the friction angle between the soil grains. The relationship can be expressed as follows:

$$
\frac{\dot{\varepsilon}_{3}}{\dot{\varepsilon}_{1}}=\frac{\sigma_{1}}{\sigma_{3} \tan ^{2}\left(45+\phi_{u} / 2\right)},
$$

where the ratio of axial stress to lateral stress at the failure state is defined as the shear failure parameter $R_{f}$ and takes form as

$$
R_{f}=\left(\frac{\sigma_{1}}{\sigma_{3}}\right)_{\max }
$$

and the failure Poisson ratio $v_{f}$ can be then obtained by $v_{f}=\left[\dot{\varepsilon}_{3} / \dot{\varepsilon}_{1}\right]_{R=R f}$ with $d_{f}=\sqrt{1+2 v_{f}^{2}}$. Fine sands are used for the reconstituted hydrate samples in this study, and the friction angle is chosen as $26^{\circ}$, and the secant modulus is adopted. At this point, as long as the hydrate saturation of GHBS is given, the 4 material parameters of the proposed constitutive model (see Equation 7) are determined and the stress-strain relationship of GHBS is eventually obtained.

\subsection{The shear strength modeled by the Mohr-Coulomb criterion (Model II)}

As a granular material, the cohesion between soil grains and hydrate is significant in GHBS. Hence, the Mohr-Coulomb criterion is adopted to study the influence of the effective confining pressure, internal friction, and cohesion. At this stage, the failure stress ratio $R_{f}$ is related to the friction angle $\phi$ as follows:

$$
R_{f}=\frac{1+\sin \phi}{1-\sin \phi}
$$

and the failure Poisson ratio $v_{f}$ can be determined by experimental data or roughly estimated with the dilatancy theory in the work of Rowe. ${ }^{22}$ Therefore, the hypoplastic model can be modified as

$$
\dot{\sigma}=c_{1}(\operatorname{tr}(\sigma-c)) \dot{\varepsilon}+c_{2} \frac{\operatorname{tr}((\sigma-c) \dot{\varepsilon})(\sigma-c)}{\operatorname{tr}(\sigma-c)}+\left(c_{3} \frac{(\sigma-c)^{2}}{\operatorname{tr}(\sigma-c)}+c_{4} \frac{\left((\sigma-c)^{*}\right)^{2}}{\operatorname{tr}(\sigma-c)}\right)\|\dot{\varepsilon}\|,
$$

where $c$ is the cohesion of GHBS.

\subsection{The hypoplastic model for the strain softening of GHBS (Model III)}

Because of the increase of the hydrate, the cementation among the grains of GHBS gets strengthened and the cohesion gets increased, and the strain softening occurs as the hydrate saturation reaches a critical extent. ${ }^{21}$ The effect of the porosity is obviously observed in various laboratory tests of soil mechanics. The preliminary physical explanation can be drawn for GHBS that as the hydrate occupies the pores of GHBS, the effective porosity decreases, and the filling of the pores and compression state leads to nontrivial rotation, slide, and rearrangement among soil grains, and the shear dilatancy occurs at the critical void ratio of GHBS. Hence, we propose a new hypoplastic constitutive model to describe the strain softening behavior as follows:

$$
\dot{\sigma}=c_{1}(\operatorname{tr}(\sigma-c)) \dot{\boldsymbol{\varepsilon}}+c_{2} \frac{\operatorname{tr}((\sigma-c) \dot{\boldsymbol{\varepsilon}})(\sigma-c)}{\operatorname{tr}(\sigma-c)}+\left(c_{3} \frac{(\sigma-c)^{2}}{\operatorname{tr}(\sigma-c)}+c_{4} \frac{\left((\sigma-c)^{*}\right)^{2}}{\operatorname{tr}(\sigma-c)}\right)\|\dot{\varepsilon}\| \cdot I_{e},
$$

where the density function $I_{e}=(a-1) D_{c}+1$ with a material constant $\left.I_{e}\right|_{e=e_{\min }}=a$ and the modified relative density $D_{c}=\left(e_{c r t}-e\right) /\left(e_{c r t}-e_{\min }\right)$, in which $e$ is the void ratio and $e_{c r t}$ and $e_{\min }$ are the critical and minimum void ratio, respectively. The void ratio $e$ can be updated during a deformation process according to the evolution equation $\dot{e}=(1+e) \operatorname{tr} D$ with a specific initial void ratio at any well-defined stress state, for example, as a function of porosity and hydrate saturation $e=\varphi\left(1-S_{h}\right) /\left(1-\varphi\left(1-S_{h}\right)\right)$. 


\section{3 | STRESS-STRAIN MEASUREMENTS OF GHBS}

\section{1 | Experimental apparatus}

Figure 1 shows the diagram of the experimental apparatus used for synthesis of GHBS and the triaxial compression tests. The apparatus is an integrated testing machine with a capacity of the effective confining pressures ranging from 0 to $14 \mathrm{MPa}$ with an accuracy of $0.5 \%$, and the temperatures can be controlled in the range of $-20^{\circ} \mathrm{C}$ to $20^{\circ} \mathrm{C}$ with an accuracy of $2.5 \%$. The apparatus can also provide a maximum backpressure by methane gas-supply cylinder from 0 to $10 \mathrm{MPa}$ for achieving the formation of methane hydrate-bearing sediments under the necessary high pressure and low temperature conditions. The total gas percolating can be measured with a gas flow meter. The GHBS can be then formed under proper pressure and temperature. More detailed information of the apparatus is referred to the previous work. ${ }^{9}$

Gas hydrate-bearing sediments are sediment of soil (sand, silt sand, clay, etc) mixed with gas, water, and gas hydrate in nature. The hydrate occurrence types are disseminated and pore-filling in coarse sands and veined or nodule in fine-grained sediments. Exploration for gas hydrate as an energy resource focuses on sandy GHBS. ${ }^{1}$ Here, sandy GHBS samples were prepared to capture the main composition and geotechnical condition.

\section{2 | Host specimen material}

The host specimen was prepared by fine silty sand, and the grain size distribution is illustrated in Figure 2 . The specific gravity is about 2.69 , and the relative density is $54 \%$. The dry density of the specimen is $1.6 \mathrm{~g} / \mathrm{cm}^{3}$ with a porosity of around $40 \%$.

\section{3 | Experimental procedure}

Gas hydrate-bearing sediments are found to distribute in permafrost and marine sediments in nature, where the temperature is above zero and the pressure is above phase equilibrium pressure of gas hydrate. The stiffness and failure strength of GHBS depend on the magnitude of temperature, and the temperature drop (from $5^{\circ} \mathrm{C}$ to $1^{\circ} \mathrm{C}$ ) leads to the increase of the stiffness and failure strength because of temperature dependence of the strength of the hydrate/sediment contact..$^{23}$ Hence, in all the tests, the temperature was kept at $1^{\circ} \mathrm{C}$ during GHBS preparation, and the triaxial compressions were conducted with little temperature change and no hydrate dissociation.

The unsaturated GHBS was prepared under the condition of partial saturated water in the pores. The gas saturated method was applied to obtain uniform gas hydrate distribution. ${ }^{23,24}$ The procedures were (1) certain water (about $20 \mathrm{~mL}$ ) was injected into the specimen. (2) The gas was supplied under the pressure of $3 \mathrm{MPa}$ and the temperature was turned to $1^{\circ} \mathrm{C}$, and the total gas volume was controlled according to the theoretical volume of reacted gas into hydrate

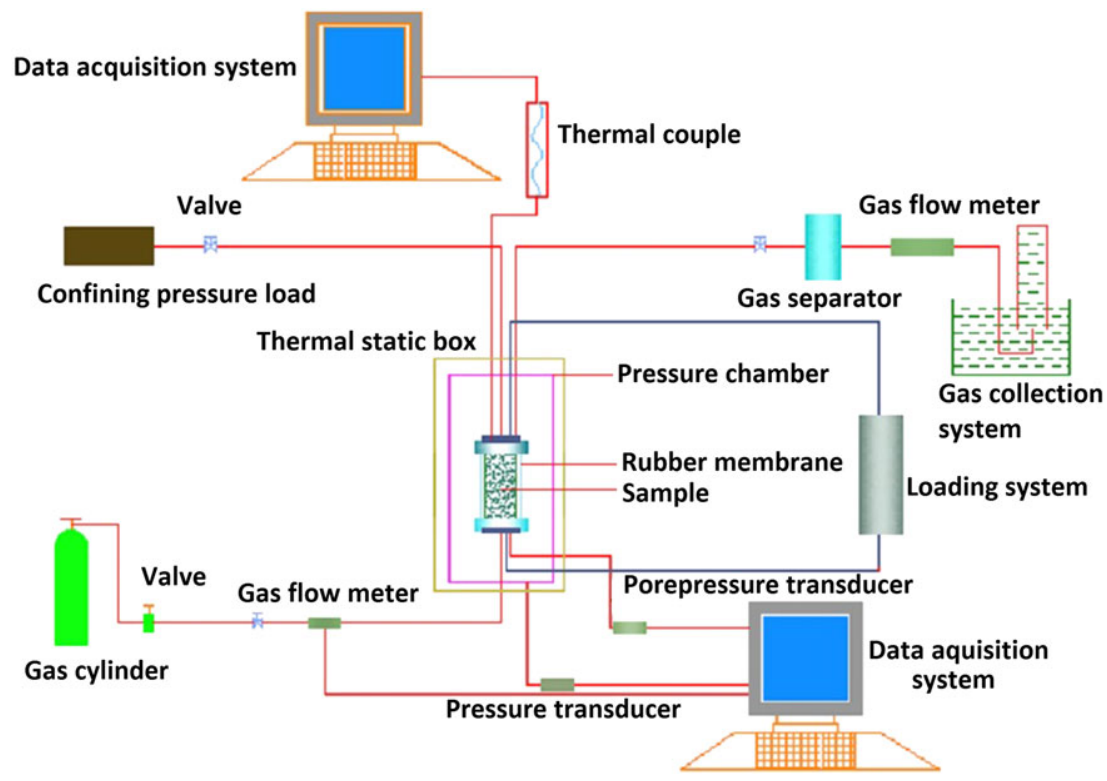

FIGURE 1 Integrated test apparatus for synthesis of gas hydrate-bearing sediment and triaxial compression tests [Colour figure can be viewed at wileyonlinelibrary. com] 


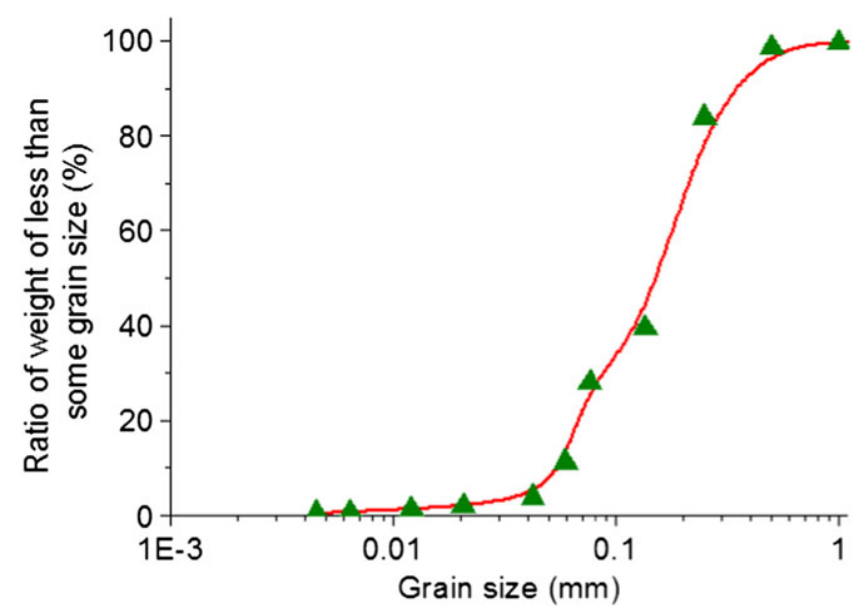

FIGURE 2 Grain size distribution of fine silty sand [Colour figure can be viewed at wileyonlinelibrary.com]

and free gas in the pores. (3) As the GHBS preparation was completed, the confining pressures were applied with 4,6 , and $8 \mathrm{MPa}$. Because of the $3 \mathrm{MPa}$ of pore gas pressure, the effective confining pressures were 1, 3, and $5 \mathrm{MPa}$ accordingly. (4) The temperature was remain to $0^{\circ} \mathrm{C}$ to $1^{\circ} \mathrm{C}$, and the average hydrate saturations were $5 \%, 11 \%, 23 \%, 40 \%$, and $52 \%$. The hydrate saturation was controlled by the methane gas pressure and the total gas volume, and the amount of gas used in GHBS formation was calculated on the basis of ideal gas equation and checked by collected gas during hydrate dissociation after triaxial tests. The mechanical properties of the reconstituted GHBS were obtained under the backpressure $3 \mathrm{MPa}$ and drained condition and listed in Table 1, such as modulus, shear strength, cohesion, and internal friction angle. The initial modulus was determined by $E=\left.(\Delta \sigma / \Delta \varepsilon)\right|_{\varepsilon=1 \%}$, and the maximum deviatoric stress was obtained until the engineering strain reached $15 \%$ (plastic failure) or the strain at failure. The cohesion and internal friction angle of GHBS with certain hydrate saturation were achieved through 3 experiments under different effective confining pressures.

\section{4 | RESULTS AND DISCUSSION}

On the basis of a series of tests, the stress-strain curves under different saturations and effective confining pressures are shown in Figure 5 (test points of stress with strain). It is noted that the stress-strain curves are obviously nonlinear and no apparent elastic parts are observed.

TABLE 1 Mechanical parameters of gas hydrate-bearing sediment

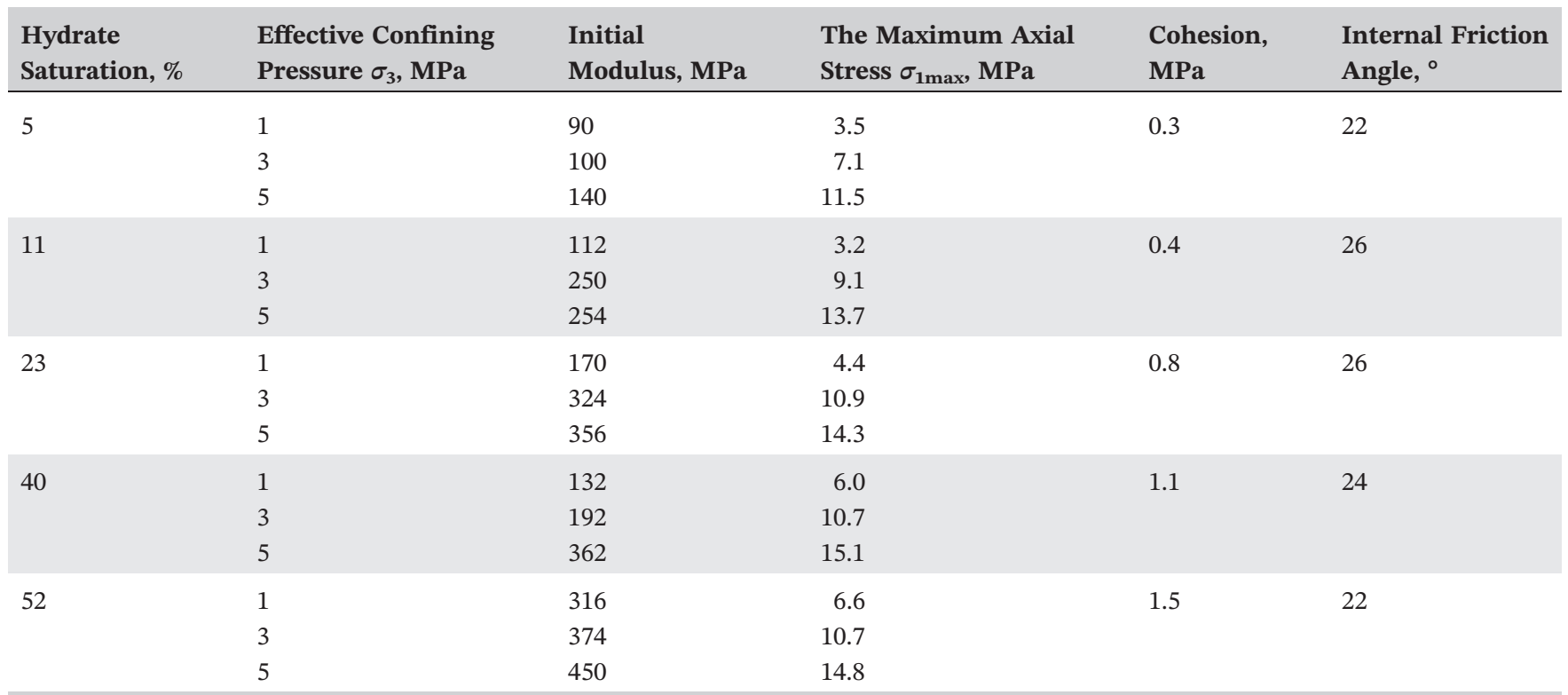




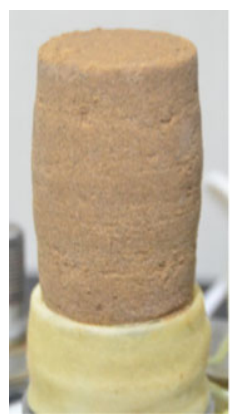

(A) hydrate saturation $11 \%$

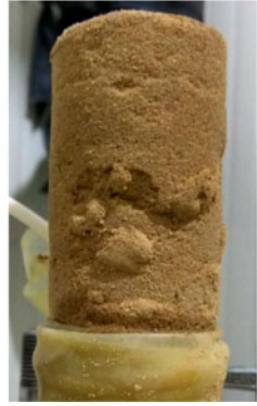

(B) hydrate saturation $52 \%$

FIGURE 3 The shape of samples after tests [Colour figure can be viewed at wileyonlinelibrary.com]

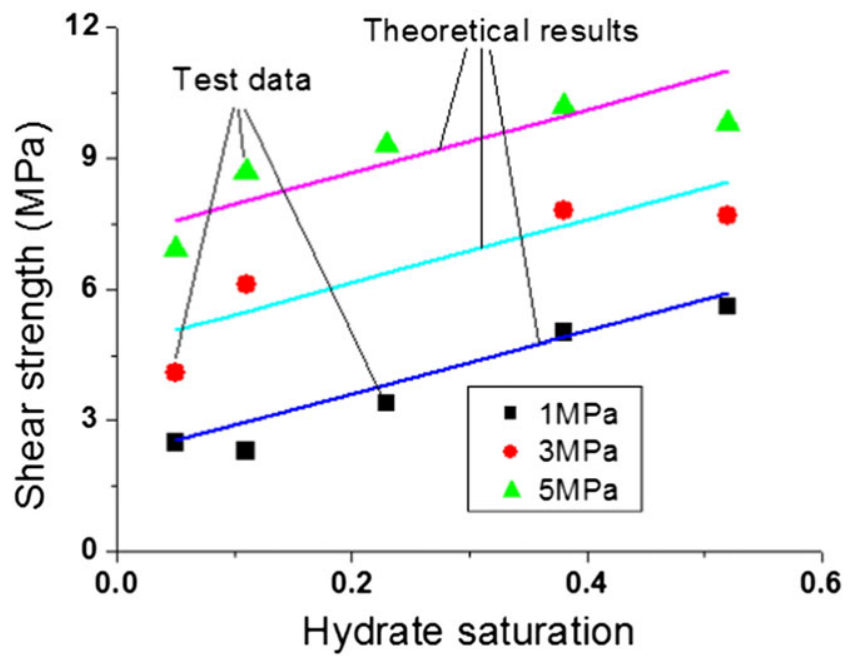

FIGURE 4 Shear strength under different hydrate saturations and effective confining pressures [Colour figure can be viewed at wileyonlinelibrary.com]

During the initial stage of compression, uniform deformation is predominant. Under low hydrate saturation $(\leq 40 \%)$, the shape of the samples changes from a cylinder into a drum-like, and the stress-strain behaves as strain hardening; under high hydrate saturation, strain softening behavior occurs, and a small shear crack at the side of the samples appears as in Figure 3. Then strain localization will occur and develop into a shear band from the crack.

To validate the proposed model for GHBS, the shear strength under different hydrate saturations and effective pressures is predicted with the proposed theoretical model using Equation 12. The parameters used in the model are

TABLE 2 Parameters used in Model I

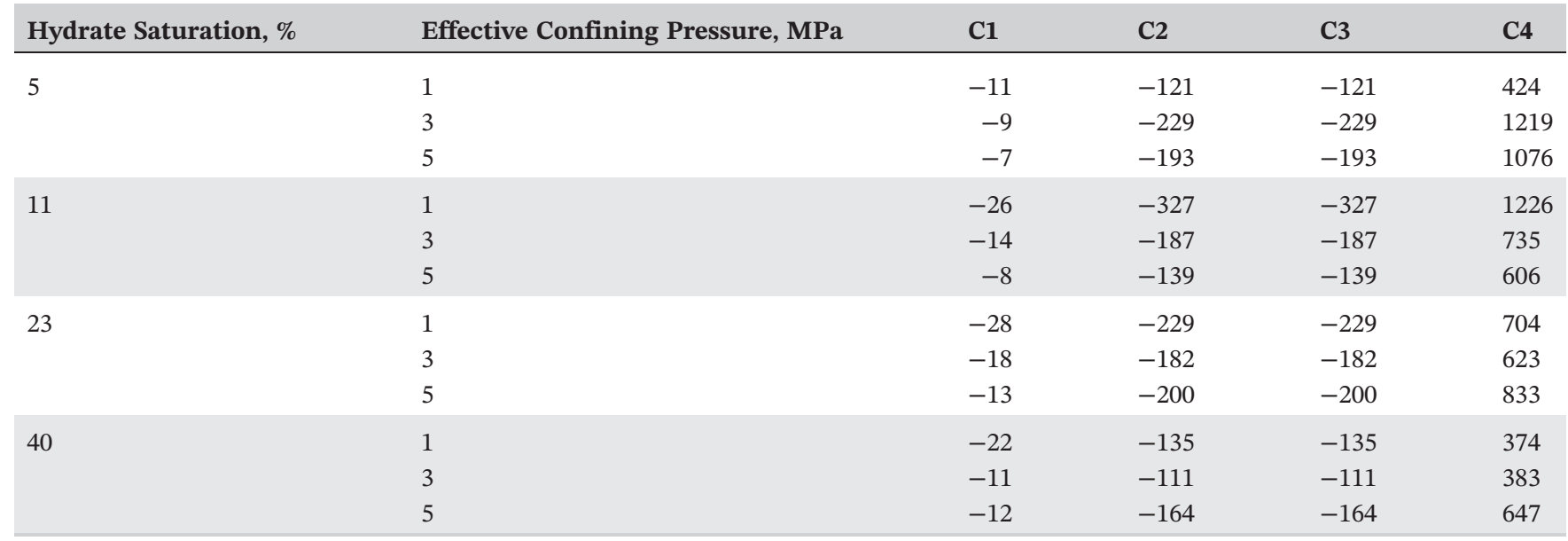



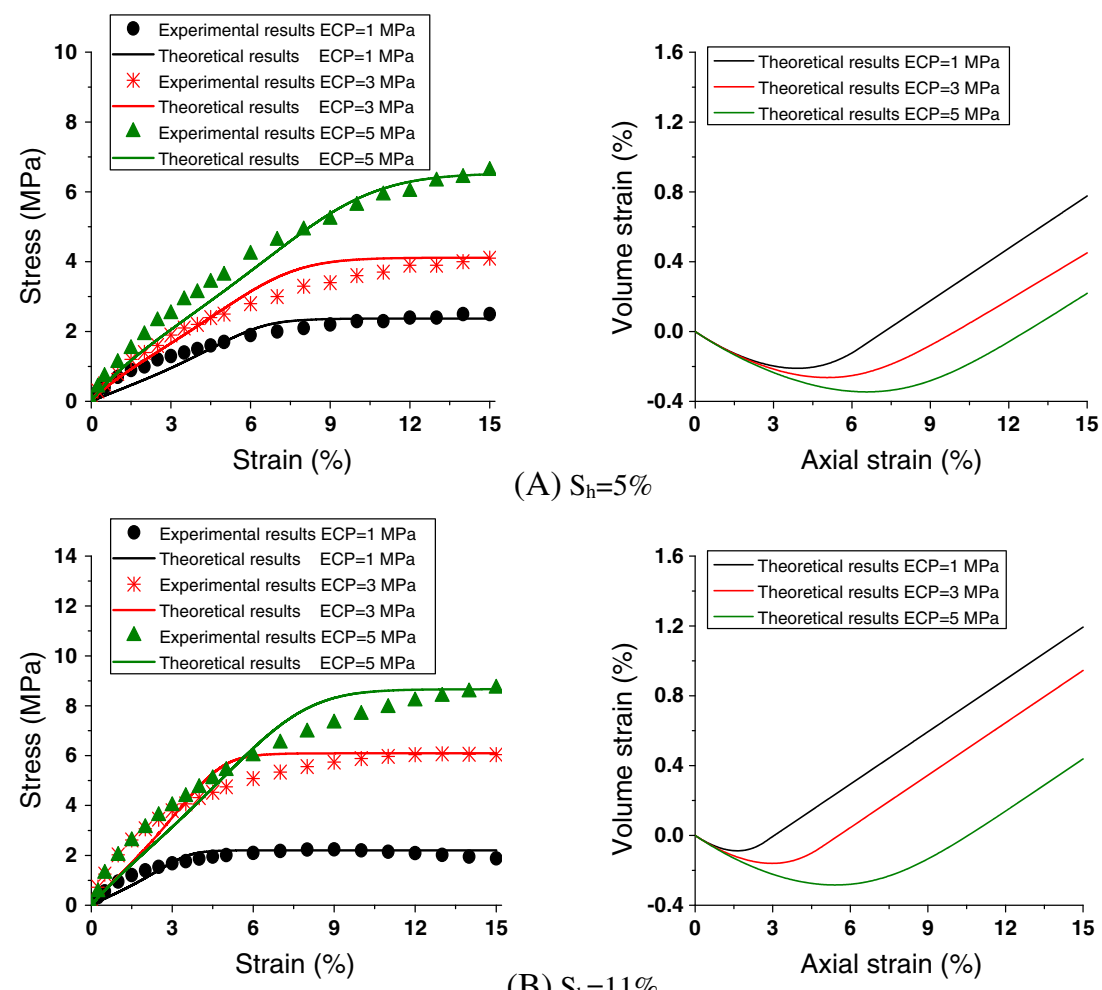

(A) $\mathrm{S}_{\mathrm{h}}=5 \%$
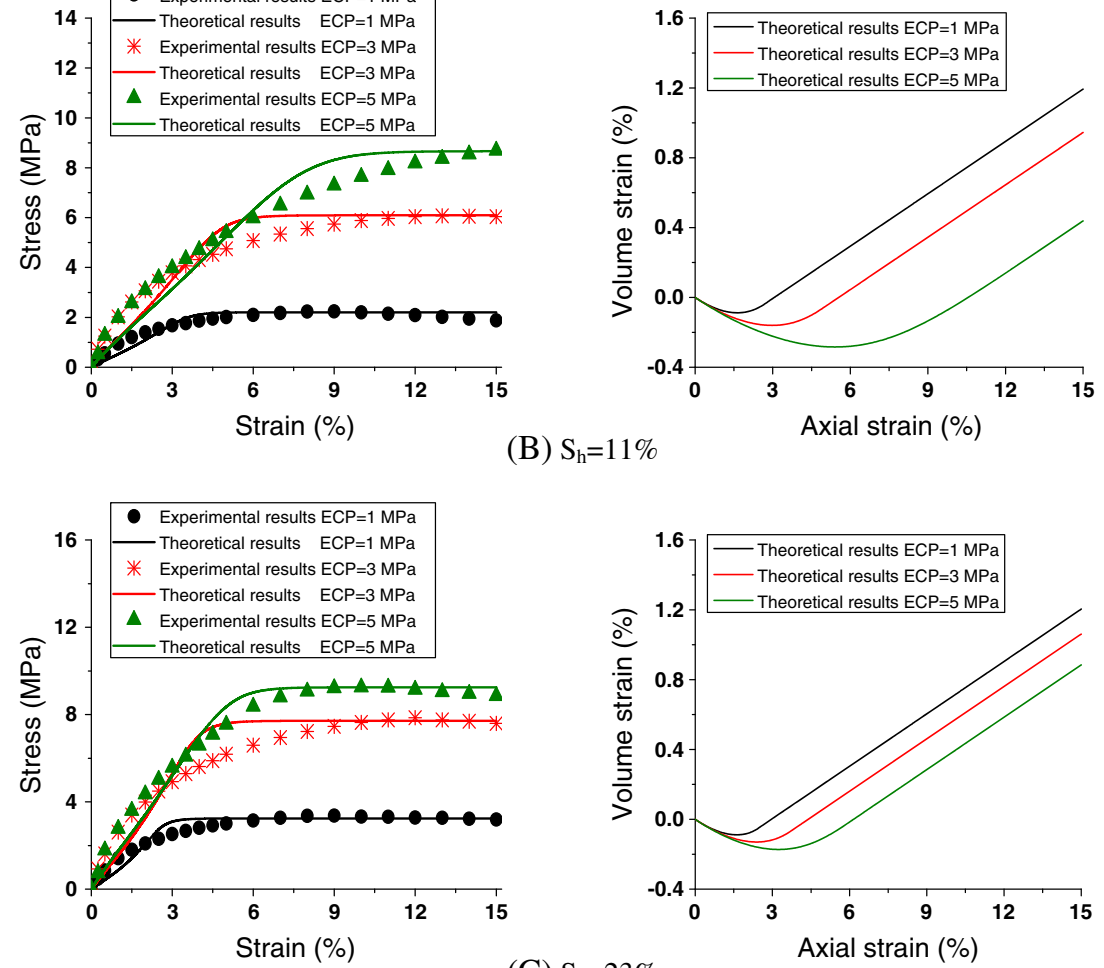

(C) $\mathrm{S}_{\mathrm{h}}=23 \%$

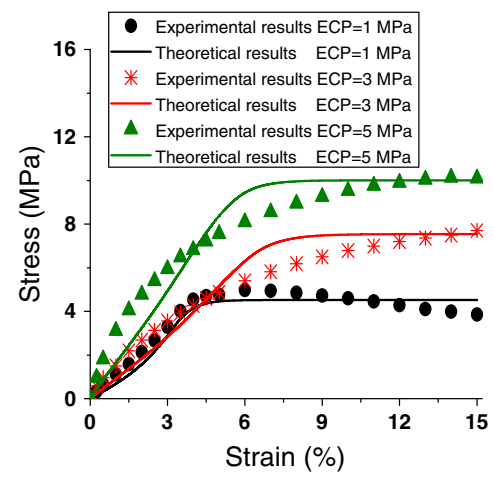
confining pressures [Colour figure can be viewed at wileyonlinelibrary.com]

FIGURE 5 Stress-strain curves under different saturations and effective

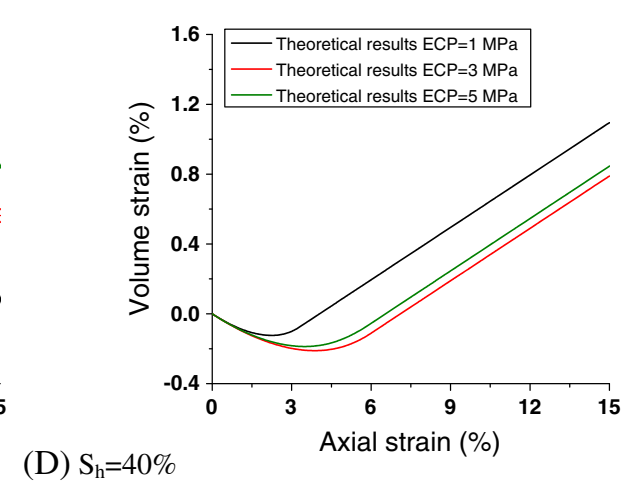

(D) $\mathrm{S}_{\mathrm{h}}=40 \%$

as follows: $A^{\prime}(0)=7.13, A(0)=0.92, B^{\prime}(0)=0.02, B(0)=1.26$, and the relative coefficient $R^{2}$ is set to be 0.92 . As shown in Figure 4 , the theoretical results are in good agreement with the test data.

The material parameters used in Model I (see in Equations 11 and 12) are then calculated according to the mechanical parameters of GHBS in Table 1 and shown in Table 2. The stress-strain curves and volume strains are simulated under the different hydrate saturations, ie, 5\%, 11\%, 23\%, and 40\%, and compared with the experimental data (see 
TABLE 3 Parameters used in Model II

\begin{tabular}{llrrrr} 
Hydrate Saturation, \% & Effective Confining Pressure, MPa & C1 & C2 & C3 & C4 \\
\hline 5 & 1 & -11 & -398 & -398 \\
& 3 & -9 & -325 & -325 \\
& 5 & -7 & -253 & -253 & 1968
\end{tabular}

Figure 5). It can be seen from the figures that the results are in good agreement with the test results and the plastic behaviors are clearly observed under low hydrate saturation, which indicates the effectiveness of the proposed Model I, while the theoretical volume change indicates that shear dilation occurs because the hypoplastic model may have a shortcoming in overestimation of dilatancy.

In Table 2, the material parameters $\mathrm{C} 2 / \mathrm{C} 1$ and $\mathrm{C} 4 / \mathrm{C} 1$ are different under different effective confining pressures, because different confining pressures lead to different failure stress ratios, and the material parameters $\mathrm{C} 2$ and $\mathrm{C} 4$ are nonlinear with the failure stress ratio.

The material parameters in Model II are calculated and listed in Table 3 according to the cohesion, internal friction angle, and hydrate saturation $S_{h}$. Under 5\% hydrate saturation, the shear strength is modeled by the Mohr-Coulomb criterion. The strain hardening behaviors under the effective confining pressures of 1,3 , and $5 \mathrm{MPa}$ are predicted and compared with the experimental results, and the theoretical volume change indicates that the shear dilation is not pronounced as shown in Figure 6.

The material parameters in Model III are calculated and listed in Table 4, and strain softening behavior is simulated under high hydrate saturation $52 \%$. The material parameters used in the experiments are as follows: Considering the effect of hydrate saturation to the effective porosity, the critical void ratio $e_{c r t}$ and the minimum void ratio $e_{\min }$ are 0.31 and 0.18 , respectively, and the parameter $a$ is set to be 0.85 . As can be seen from Figure 7 that the stress-strain results obtained by Model III are in good agreement with the experimental results. The important characteristics of strain softening behavior are well predicted under high hydrate saturation (52\%), the volume strain decreases with the increase of the effective confining pressure, and the theoretical volume change indicates that the shear dilation is more pronounced than of that lower hydrate saturation.

For hydrate saturation 5\%, the critical void ratio $e_{c r t}$ and the minimum void ratio $e_{\min }$ are 0.42 and 0.70 , respectively, and the parameter $a$ is set to be 0.85 . The material parameters $\mathrm{C} 1 \sim \mathrm{C} 4$ are adopted in Table 2 . As can be seen from
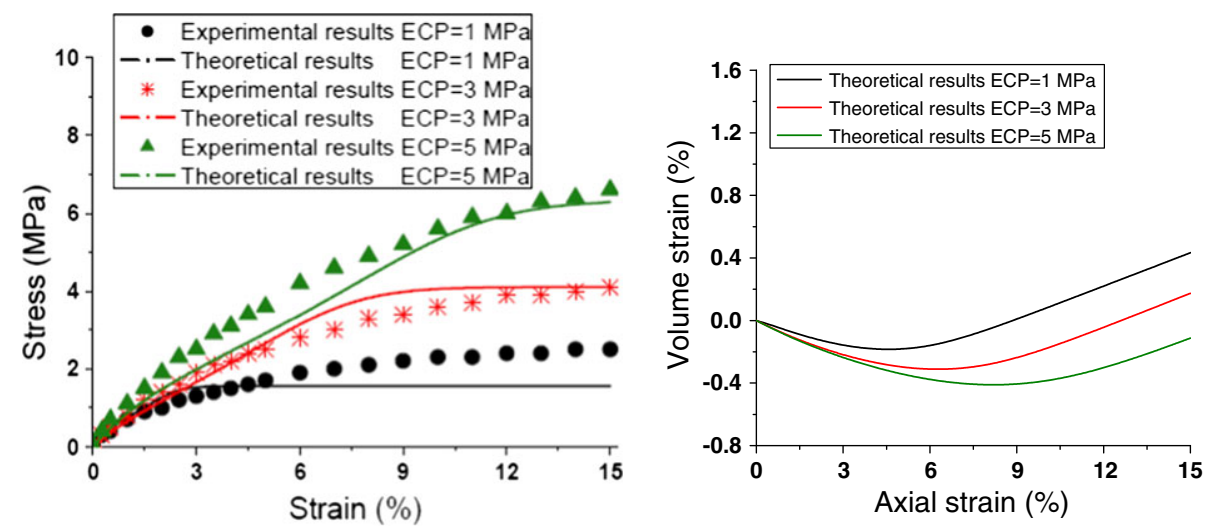

FIGURE 6 Stress-strain curves under the hydrate saturation of 5\% in Model II [Colour figure can be viewed at wileyonlinelibrary.com]

TABLE 4 Parameters used in Model III

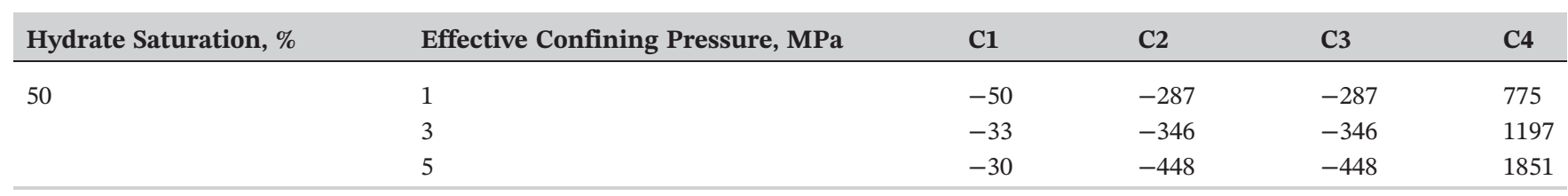


FIGURE 7 Stress-strain curves and shear dilation in Model III $\left(\mathrm{S}_{\mathrm{h}}=52 \%\right)$ [Colour figure can be viewed at wileyonlinelibrary.com]

FIGURE 8 Stress-strain curves and volume strain in Model III $\left(\mathrm{S}_{\mathrm{h}}=5 \%\right)$ [Colour figure can be viewed at wileyonlinelibrary.com]

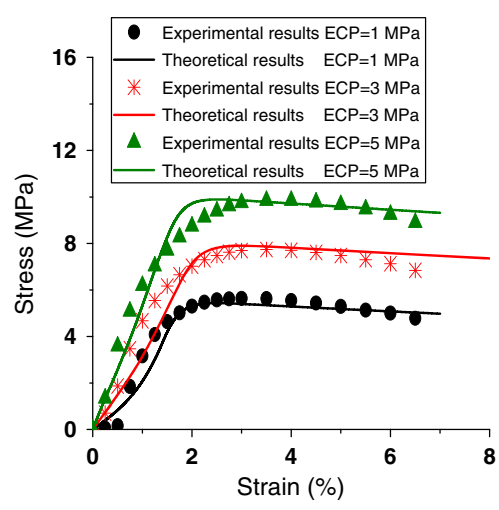

(A) Stress-strain curve

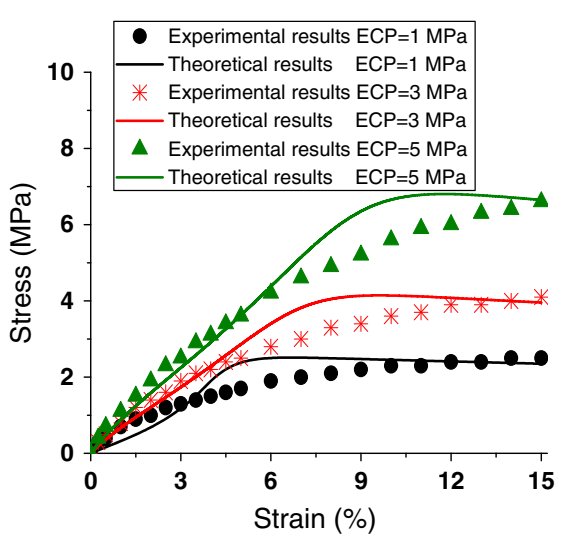

(A) Stress-strain curve

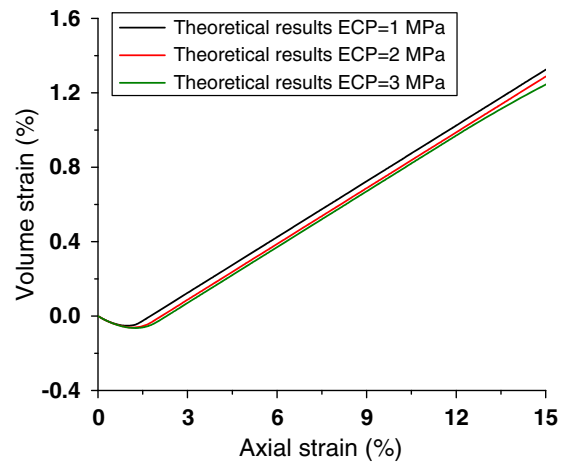

(B) Volume strain

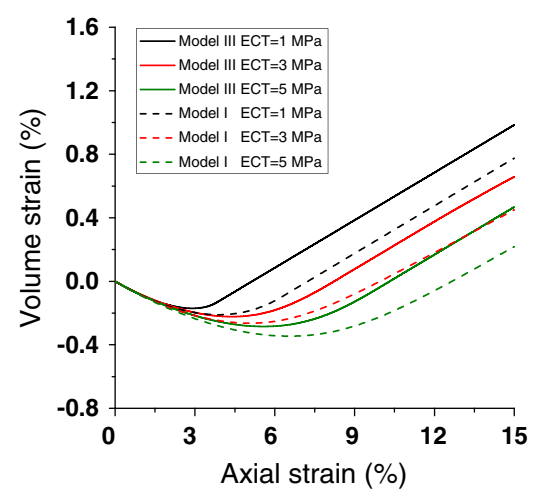

(B) Volume strain

Figure 8 that the stress-strain results obtained by Model III reach the peak shear strengths in lower strains than those of tests. The theoretical volume strains are larger than that by Model I.

\section{5 | CONCLUSIONS}

The mechanical properties of GHBS are investigated by the integrated experimental apparatus with triaxial compression tests and compared with the simulated results obtained by the proposed hypoplastic models. The Drucker-Prager criterion and the Mohr-Coulomb criterion are introduced into the hypoplastic model to analyze the shear strength with considering the effective porosity because of the hydrate filling in the pores of GHBS. The effects of the hydrate saturation and the effective confining pressures are studied with both experimental apparatus and the proposed hypoplastic model, and the comparison shows that the proposed hypoplastic model is capable of capturing the salient features of the mechanical properties of GHBS. Moreover, the proposed model is verified to be able to describe both the strain hardening behavior under low hydrate saturation $(\leq 40 \%)$ and the strain softening under high hydrate saturation $(>40 \%)$ of GHBS. It is also worth noting that the empirical and experimental parameters of the model are much less than the classical elastoplastic models, and their physical meanings can be clearly related to the well-established models. The proposed model is verified to be a simple and practical reference for the evaluation of soil deformation and failure behaviors in hydrate exploration and exploitation.

\section{ACKNOWLEDGEMENTS}

This study is part of project nos. 41376078 and 51639008 funded by the National Natural Science Foundation of China, project no. DD20160216 funded by China Geological Survey, open research fund program of State Key Laboratory of Hydroscience and Engineering, Tsinghua University (sklhse-2017-D-02), and Youth Innovation Promotion Association of the Chinese Academy of Sciences (2017027). 


\section{ORCID}

Xuhui Zhang (D) http://orcid.org/0000-0001-9614-3679

\section{REFERENCES}

1. Waite WF, Santamarina JC, Cortes DD, et al. Physical properties of hydrate-bearing sediments. Rev Geophys, RG4003/2009. 2009;47:1-38.

2. Kwon TH, Oh TM, Choo YW, Lee C, Lee KR, Cho GC. Geomechanical and thermal responses of hydrate bearing sediments subjected to thermal simulation: physical modeling using a geotechnical centrifuge. Energy Fuel. 2013;27(8):4507-4522.

3. Kimoto S, Oka F, Fushita T. A chemo-thermo-mechanically coupled analysis of ground deformation induced by gas hydrate dissociation. Int J Mech Sci. 2010;2010(52):365-376.

4. Yoneda J, Masui A, Konno Y, et al. Mechanical properties of hydrate-bearing turbidite reservoir in the first gas production test site of the eastern Nankai trough. Mar Pet Geol. 2015;66(2):471-486.

5. Hyodo M, Li YH, Yoneda J, Nakata Y, Yoshimoto N, Nishimura A. Effects of dissociation on the shear strength and deformation behavior of methane hydrate-bearing sediments. Mar Pet Geol. 2014;51:52-62.

6. Miyazaki K, Masui A. Tri-axial compressive properties of artificial methane hydrate bearing sediment. J Geophys Res. 2011;116:B06102

7. Winters WJ, Pecher IA, Waite WF, Mason DH. Physical properties and rock physics models of sediment containing natural and laboratoryformed methane gas hydrate. Am Mineral. 2004;89(8-9):1221-1227.

8. Winters WJ, Waite WF, Mason DH, Gilbert LY, Pecher IA. Methane gas hydrate effect on sediment acoustic and strength properties. J Petrol Sci Eng. 2007;56(1-3):127-135.

9. Zhang XH, Lu XB, Zhang LM, Wang SY, Li QP. Experimental study on mechanical properties of methane-hydrate-bearing sediments. Acta Mech Sin. 2012;28(5):1356-1366.

10. Zhang XH, Lu XB, Shi YH, Xia Z. Study on the mechanical properties of hydrate-bearing silty clay. Mar Pet Geol. 2015b;67:72-80.

11. Pinkert S, Grozic JLH. Experimental verification of a prediction model for hydrate-bearing sand. J Geophys Res Solid Earth. 2016;121(6):4147-4155.

12. Pinkert S, Grozic JLH, Priest JA. Strain-softening model for hydrate-bearing sands. Int J Geomech. 2015;15(6):04015007

13. Zhang XH, Lu LL, Zhou JB, et al. A model for the elastic modulus of hydrate-bearing sediments. Int J Offshore Polar Eng. 2015a;25(4):314-319.

14. Li YH, Song YC, Liu W, Liu WG, Yu F, Wang R, Nie XF. Analysis of mechanical properties and strength criteria of methane hydrate-bearing sediments. Int J Offshore Polar Eng. 2012;22(4):290-296.

15. Miyazaki K, Tenma N, Aoki K, Yamaguchi T. A nonlinear elastic model for triaxial compressive properties of artificial methane-hydratebearing sediment samples. Energies. 2012;5(12):4057-4075.

16. Yu F, Song YC, Liu WG, Li YH, Lam W. Analyses of stress strain behavior and constitutive model of artificial methane hydrate. J Petrol Sci Eng. 2011;77(2):183-188.

17. Jiang MJ, Sun YG, Yang QJ. Simple distinct element modeling of the mechanical behavior of methane hydrate-bearing sediments in deep seabed. Granular Matter. 2013;15(2):209-220.

18. Lin J, Wu W, Borja R. Micropolar hypoplasticity for persistent shear band in heterogeneous granular materials. Comput Methods Appl Mech Eng. 2015;289:24-43.

19. Lin J, Wu WA. Comparative study between DEM and micropolar hypoplasticity. Powder Technol. 2016;293:121-129.

20. Wu W, Bauer EA. Simple hypoplastic constitutive model for sand. Int J Numer Anal Methods Geomech. 1994;18(12):833-862.

21. Wu W, Bauer E, Kolymbas D. Hypoplastic constitutive model with critical state for granular materials. Mech Mater. 1996;23(1):45-69.

22. Rowe PW. The stress-dilatancy relation for static equilibrium of an assembly of particles in contact. Proceedings of the Royal Society A. 1962;264:500-527.

23. Hyodo M, Li YH, Yoneda J, et al. Mechanical behavior of gas-saturated methane hydrate-bearing sediments. J Geophys Res Solid Earth. 2013;118(10):5185-5194.

24. Liu, L.L. Evolution of gas hydrate dissociation front in hydrate bearing sediment. Ph.D. Thesis, Institute of Mechanics, Chinese Academy of Sciences, Beijing, 2013

How to cite this article: Zhang X, Lin J, Lu X, et al. A hypoplastic model for gas hydrate-bearing sandy sediments. Int J Numer Anal Methods Geomech. 2018;42:931-942. https://doi.org/10.1002/nag.2772 